\title{
Ein EMG-Algorithmus differenziert verlässlich
}

Fragestellung: Die Autoren stellen eine durch gleichzeitiges Video-EEG validierte prospektive Studie vor, bei der ein automatisierter EMG-Algorithmus zur Differenzierung zwischen konvulsiven epileptischen und psychogenen nicht-epileptischen Anfällen eingesetzt wurde.

Hintergrund: Ein wesentliches Problem der klinischen Praxis in der Epileptologie stellt die Differenzialdiagnose zwischen psychogenen und epileptischen Anfällen dar. Oft ist dies nur möglich mit zeit- und personalaufwendigem Langzeit-Video-EEG. Die dänische Arbeitsgruppe hat einen automatisierten EMG-Algorithmus entwickelt, der sich die typische hochfrequente EMG-Aktivität während bilateral konvulsiver (gemäß alter Klassifikation generalisiert tonisch-klonischer) Anfälle zu Nutze macht, diesen in einer prospektiven Studie in der klinischen Praxis eingesetzt und auf seine diagnostische

Beniczky S, Conradsen I, Moldovan $\mathrm{M}$ et al. Automated differentiation between epileptic and nonepileptic convulsive seizures. Ann Neurol 2015; 77: 348-51
Qualität hin untersucht.

Patienten und Methodik: 24 konsekutive Patienten mit offener Differenzialdiagnose bezüglich psychogener oder epileptischer Anfälle wurden in einer Epilepsie-MonitoringEinheit mittels Langzeit-Video-EEG und dem automatisierten EMG-Algorithmus untersucht. Insgesamt wurden 44 Anfälle erfasst, von denen 25 epileptischen Anfällen bei elf Patienten und 19 nicht-epileptischen Anfällen bei 13 Patienten entsprachen. Goldstandard für die diagnostische Klassifikation war das Langzeit-Video-EEG, das von einem erfahrenen Epileptologen ausgewertet wurde, der für die Ergebnisse des EMG-Algorithmus verblindet war. Danach wurden Sensitivität, Spezifität und diagnostische Präzision (Accuracy) ausgewertet.

Ergebnisse: Der Algorithmus klassifizierte 96\% der epileptischen bilateral konvulsiven Anfälle und $95 \%$ der nicht-epileptischen Anfälle korrekt. Die diagnostische Präzision betrug 95\%. Ein bilateral konvulsiver Anfall wurde nicht erfasst. Zwei nicht-epileptische Anfälle wurden zu Unrecht als epileptisch klassifiziert.

Schlussfolgerungen: Die Autoren kommen zu dem Schluss, dass der Algorithmus sich als geeignet dafür erwies, zwischen epileptischen und nicht-epileptischen konvulsiven Anfällen zu differenzieren.

\section{- Kommentar von Bernhard J. Steinhoff, Kehl-Kork}

\section{Das eigentliche klinisch relevante Problem wurde nicht adressiert}

Es ist sicherlich verdienstvoll, einfache Methoden zu entwickeln, die die Differenzierung zwischen epileptischen und nicht-epileptischen Anfällen erleichtern. Hierzu gibt es seit vielen Jahren verschiedene Ansätze, die von den Autoren auch teilweise zitiert werden und zu denen beispielsweise laborchemische (Kreatinkinase, Prolaktin), akzelerometrische oder kardiologische (Herzratenvariabilität) Ansätze gehören.

Dass ein automatisierter EMG-Algorithmus geeignet ist, zwischen großen epileptischen Anfällen und nicht-epileptischen psychogenen Anfällen zu differenzieren, überrascht nicht. In Zeiten alter Krankenhausbetten war der erfahrene Neurologe in der Lage, schon am Geräusch des Bettes im Anfall die entsprechende Differenzierung sicher vorzunehmen, weil ein bilateral konvulsiver epileptischer Anfall völlig anderen rhythmischen Gegebenheiten unterliegt als der psychogene Anfall mit seiner typischen wechselnden und fluktuierenden motorischen Rhythmik. Aus Sicht des Kommentators ist es eher überraschend, dass bei der hier untersuchten Fragestellung überhaupt falsche Zuordnungen möglich waren. Das differenzialdiagnostische Problem besteht eigentlich nämlich nicht dann, wenn Patienten psychogene oder epileptische konvulsive Anfälle haben, sondern wenn es um die Differenzialdiagnose zwischen psychogenen und epileptischen hypermotorischen oder dyskognitiv fokalen Anfällen geht. Hierauf verweisen die Autoren auch in der Diskussion ausdrücklich und bedauern, dass ihre Methode nicht geeignet ist, hypermotorische epileptische Anfälle zu erkennen. Insofern ist zu bezweifeln, ob - wie die Autoren als klinische Perspektive diskutieren - ein wirklich sinnvoller nächster Schritt der Einsatz tragbarer Oberflächen-EMG-Geräte bei Ambulanzpatienten sein könnte. Erfahrungsgemäß ist die Anfallsfrequenz der Patienten, bei denen zwischen bilateral konvulsiven epileptischen und psychogenen konvulsiven Anfällen unterschieden werden muss, nicht sehr hoch. Vermutlich ist es billiger und treffsicherer, Angehörige und Augenzeugen zu bitten, bei Anfällen mittels Smartphones Videoaufzeichnungen des Anfallsgeschehens festzuhalten als auf die hier vorgestellte EMG-Methodik zurückzugreifen. Trotzdem ist die Studie sicherlich methodologisch gut sowie gewissenhaft durchgeführt und liefert interessante Ergebnisse. Allerdings - dies als abschließendes Fazit - ist das eigentliche klinisch relevante differenzialdiagnostische Problem hier nicht adressiert.

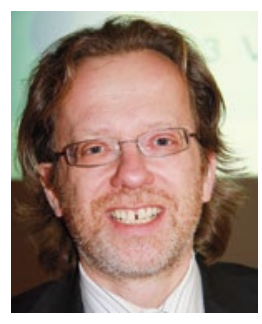

Prof. Dr. med. Bernhard J. Steinhoff, Kehl-Kork

Ärztlicher Direktor, Epilepsiezentrum Kork, Kehl-Kork

E-Mail: bsteinhoff@epilepsiezentrum.de 